\title{
Termine
}

Institut für Weiterbildung, Beratung und Planung im sozialen Bereich e.V.

Fachtagung für SupervisorInnen

„Supervision - Systemkompetenz im ostdeutschen Kontext“

23.-24. Juni 2000, Jena

Leitung: Heinrich Fallner, Prof. Dr. Wolfgang Kühl, Ruth Weismann

Information und Anmeldung: IWIS, Carl-Zeiss-Str. 2, 07743 Jena, Fax 03641/930401.

29. Internationaler Sommerkurs Berlin

,Tradition und Wandel in Gruppen und Organisationen“

15.-25.8.2000, Europäische Akademie Berlin

Ein Kooperationsangebot des Deutschen Arbeitskreises für Gruppenpsychotherapie und Gruppendynamik e.V. (DAGG), der Deutschen Gesellschaft für Supervision (DGSv) und der Europäischen Akademie Berlin.

Information und Anmeldung: Europäische Akademie Berlin, Bismarckallee 46-48, 14193 Berlin.

21. Jahrestagung der Deutschen Arbeitsgemeinschaft für Familientherapie (DAF)

in Kooperation mit dem Institut für Sozialpädagogik der FU Berlin und dem Dachverband für Familientherapie und Systemisches Arbeiten (DFS)

„Prinzip Hoffnung. Systemische Perspektiven bei Armut, Devianz, Sucht, Gewalt und Krankheit“"

20.9.-23.9.2000, Berlin

Information und Anmeldung: DAF-Jahrestagung, Context-Institut für Beratung, Dr. Marie-Luise Conen, Lauenburger Str. 37, 12169 Berlin, Tel. 030/79784087, Fax 030/7954717.

\section{Bundeskongress Soziale Arbeit}

„Die Perspektiven der Sozialen Arbeit im europäischen Integrationsprozess“

20.-22.9.2001, Mainz

veranstaltet vom Initiativkreis Bundeskongress Soziale Arbeit

Organisationsbüro: Pädagogisches Institut der Johannes Gutenberg Universität Mainz, 55099 Mainz, Tel. 06131/392918, Fax 06131/395995. 